\title{
Leukocyte transmigration requires kinesin-mediated microtubule-dependent membrane trafficking from the lateral border recycling compartment
}

\author{
Zahra Mamdouh, ${ }^{1}$ Geri E. Kreitzer, ${ }^{2}$ and William A. Muller $^{1}$
}

'Department of Pathology and Laboratory Medicine and the Graduate Program in Immunology and Microbial Pathogenesis and ${ }^{2}$ Department of Cellular and Developmental Biology, Weill Cornell Medical College, New York, NY 10021

Diapedesis of leukocytes across endothelial cells is a crucial step in both the innate and adaptive immune responses. Surface molecules on leukocytes and endothelial cells critical for diapedesis have been identified, but the mechanisms underlying this process are not understood. Homophilic interaction between platelet/endothelial cell adhesion molecule (PECAM) on leukocytes and PECAM at the endothelial border triggers targeted recycling of membrane from a reticulum localized close to the endothelial cell lateral border. This membrane surrounds the transmigrating leukocyte (Mamdouh, Z., X. Chen, L.M. Pierini, F.R. Maxfield, and W.A. Muller. 2003. Nature. 421:748-753). How this process occurs and whether it is required for diapedesis independent of PECAM are not known. We now report that targeted recycling from this lateral border recycling compartment (LBRC) is required for diapedesis, is mediated by kinesin family molecular motors, and requires normally functioning endothelial microtubules. Selective disruption of microtubules or inhibition of kinesin motor domain blocked targeted recycling and diapedesis of monocytes. Furthermore, targeted recycling of membrane from the LBRC was required for transmigration of lymphocytes, which migrate independently of PECAM. Thus, trafficking of membrane from the LBRC to surround leukocytes may be a general requirement for migration of leukocytes across the endothelial cell border. Furthermore, these data provide the first demonstration of a role for endothelial microtubules and kinesins in promoting diapedesis, and a mechanism to explain targeted recycling.

CORRESPONDENCE

William A. Muller:

wamuller@northwestern.edu

Abbreviations used: DCN, demecolcine (colcemide); HRP, horseradish peroxidase; $\mathrm{HU}-$

VEC, human umbilical vein endothelial cell; ICAM, intercellular adhesion molecule; immuno-EM, immunoelectron microscopy; LBRC, lateral border recycling compartment; PECAM, platelet/endothelial cell adhesion molecule; TEM, transendothelial migration; VE, vascular endothelial cell.
Both the innate and adaptive immune responses are dependent on the migration of leukocytes across endothelial cells. Most of this cellular traffic, or transendothelial migration (TEM), takes place at endothelial cell borders by the process of diapedesis, whereby leukocytes squeeze in ameboid fashion between the tightly apposed endothelial cells (1). Although the surface molecules used by leukocytes and endothelial cells during diapedesis have been well characterized, the mechanisms by which they regulate this process are poorly understood $(1,2)$.

Platelet/endothelial cell adhesion molecule 1 (PECAM; also known as CD31) is a transmembrane glycoprotein expressed on platelets and most leukocytes, and concentrated at the

W.A. Muller's present address is Dept. of Pathology, Northwestern University Feinberg School of Medicine, Chicago, IL 60611

The online version of this article contains supplemental material. borders of endothelial cells $(3,4)$. Homophilic interaction between PECAM on the leukocyte and PECAM at the endothelial cell border is required for diapedesis to occur in vitro and in vivo under many, but not all, inflammatory conditions $(5-8)$. We recently reported that approximately one third of total cellular PECAM exists in a membrane reticulum located below the surface of the cell at the lateral borders (9). This membrane reticulum is composed of interconnected 50-nm vesicle-like structures proximal to and contiguous with the lateral plasma membrane. These are distinct from previously described vesiculo-vacuolar organelles (10) and caveolae (9). In resting endothelial cells, membrane traffics constitutively between the lateral cell border and this compartment, which we refer to as the lateral border recycling compartment (LBRC), recycling uniformly along the lateral border with a $t_{1 / 2}$ of $\sim 10 \mathrm{~min}$. However, 
when a neutrophil or monocyte transmigrates across the endothelial cell, recycling PECAM-bearing membrane is targeted rapidly and extensively to the site of diapedesis (9). Targeted recycling of membrane from the LBRC to surround a transmigrating leukocyte requires homophilic PECAMPECAM interactions at the apical side of the cell border. If this interaction is inhibited, both TEM and targeted recycling are blocked (9). However, the factors that regulate targeted recycling are not known.

Because the structure of the LBRC and its trafficking are distinct from those of other known endocytic systems, it is unclear how the membrane is redirected to surround transmigrating leukocytes. Furthermore, it is unclear whether targeted recycling of membrane from this compartment by itself is required for transmigration, independent of PECAM. Because microtubules are involved in directed transport of vesicles over relatively long distances (11), we examined the role of microtubules in targeted recycling of membrane from the LBRC to sites of diapedesis. Using a nonblocking PECAM antibody to mark the recycling compartment, we show that membrane from the LBRC is targeted to the site of diapedesis in a microtubule-dependent manner. Furthermore, LBRC membrane targeting to sites of diapedesis is dependent on kinesin-mediated, microtubule-based transport. Depolymerization of microtubules or inhibition of kinesin blocks both targeted recycling of membrane from the LBRC and transmigration of leukocytes both under conditions in which TEM is PECAM dependent and under conditions in which it is PECAM independent.

\section{RESULTS}

We first determined conditions for depolymerizing microtubules in confluent endothelial monolayers that had no detectable effect on endothelial junctional integrity. We treated confluent monolayers of human umbilical vein endothelial cells (HUVECs) with $10 \mu \mathrm{M}$ of the microtubule-depolymerizing drug demecolcine (DCN; also known as colcemide) for 1 h. Fig. 1 shows z-series confocal projections of HUVECs stained with anti-tubulin and anti-PECAM or anti-vascular endothelial cell (VE)-cadherin antibodies. Untreated cells show well-defined radial arrays of microtubules. In contrast, microtubules were almost completely depolymerized in cells treated with DCN, allowing extraction of soluble tubulin monomers (Fig. 1 A). The monolayers remained intact and endothelial borders were not affected by this treatment, as shown by the continuous staining of PECAM and VE-cadherin at the cell borders in both control and DCN-treated cells (Fig. 1 A). Thus, microtubule disruption does not affect endothelial cell border integrity by this criterion.

We also examined whether treatment with DCN caused a functional change in junctional integrity by measuring the permeability of these monolayers to albumin over a time course relevant to our experiments. Endothelial monolayers were treated with DCN, as described in the previous paragraph, and incubated with rhodamine-labeled albumin. After $1 \mathrm{~h}$, the monolayers were washed briefly, and the albu- min that had leaked across into the collagen was quantified using a fluorescent plate reader. There was no significant difference in albumin flux across DCN-treated monolayers compared with controls (control and DCN = 3,694 \pm 787 and 4,077 \pm 104 arbitrary fluorescence units, respectively; $\mathrm{P}=0.24)$, whereas insudation into bare collagen gels was significantly higher $(8,177 \pm 193$ arbitrary fluorescence units; $\mathrm{P}=9.3 \times 10^{-5}$ vs. control; $\mathrm{P}=1.4 \times 10^{-8}$ vs. $\left.\mathrm{DCN}\right)$. Furthermore, DCN treatment did not alter the total expression of PECAM on the endothelial surface (i.e., at the cell borders), as assessed by flow cytometric analysis of isolated cells (Fig. $1 \mathrm{~B}$ ).

\section{Depolymerizing microtubules blocks targeted recycling and TEM}

As described in the previous section, PECAM and VE-cadherin localization at cell borders were not affected by DCN treatment. However, TEM was inhibited by $85 \%$ when endothelial monolayers were pretreated with $10 \mu \mathrm{M}$ DCN (Fig. 2 A). This was not caused by the effects of DCN on monocyte adhesion, because adhesion was similar in control and DCNtreated cells (in a typical experiment, adherence was $48.5 \pm 10$ and $46.5 \pm 6$ monocytes/high power field for controls and DCN-treated monolayers, respectively). The magnitude of this block was similar to that observed previously with antiPECAM blocking antibodies or soluble PECAM-Fc chimeras $(5,7,12)$. Indeed, when compared directly with anti-PECAM $\mathrm{mAb}$ in the same experiment, DCN blocked TEM to the same extent as anti-PECAM mAb (Fig. 2 B). Moreover, the two treatments were not additive, suggesting that they work at the same step in TEM. Control experiments (eluate control; see Materials and methods for a complete description) demonstrated there was no carryover of residual DCN to the leukocytes (Fig. 2 B).

When we examined recycling from the LBRC during monocyte diapedesis, control monolayers showed a bright ring-like zone of fluorescence representing recycled membrane surrounding monocytes caught in the act of diapedesis, as previously described (9). In stark contrast, such enrichment was never seen in the DCN-treated monolayers (Fig. 3). It is important to reiterate that the tag used to track recycling PECAM is an Fab fragment of a nonblocking antibody that does not interfere with any known PECAM function (6). It serves merely as a marker for membrane flow from the LBRC. DCN treatment did not inhibit adhesion of monocytes to the endothelial cells (see the previous paragraph); monocytes adhered and crawled to the junctions where they accumulated but were unable to cross the monolayer (Fig. 3, $A$ and $B$, left).

We quantified enrichment of the LBRC at sites of leukocyte-endothelial cell interaction in the presence or absence of DCN by measuring recycled PECAM fluorescence intensity (see Materials and methods), as demonstrated in Fig. 3 C. In contrast to the distinct zones of enrichment of recycling LBRC membrane seen around leukocytes in control monolayers, on DCN-treated monolayers we usually saw monocytes arrested 


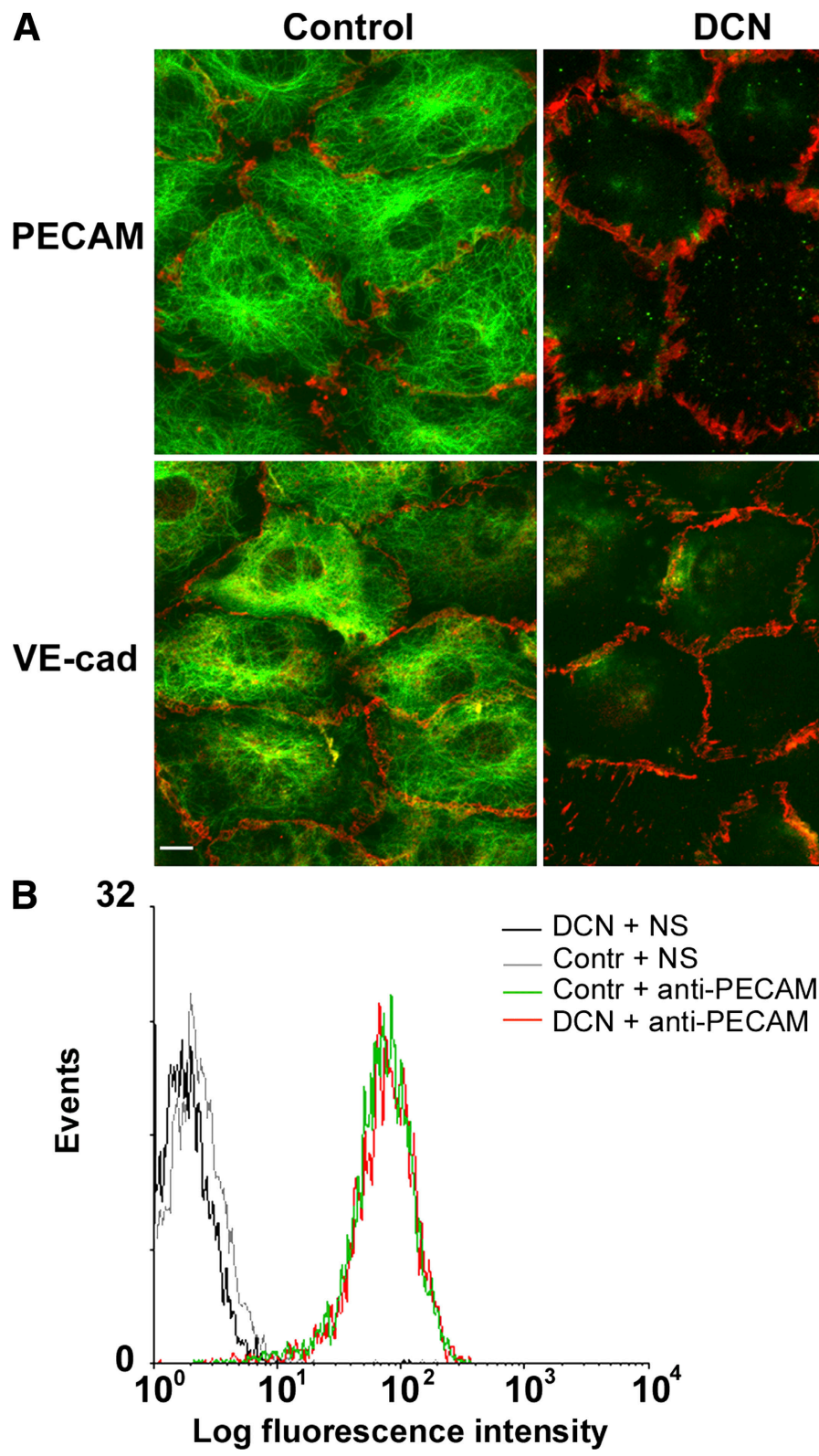

Figure 1. DCN treatment of HUVECs depolymerizes microtubules without disrupting intercellular junctions or expression of PECAM or VEcadherin. (A) HUVEC monolayers were incubated in the presence of DMSO (vehicle control) or $10 \mu \mathrm{M} \mathrm{DCN}$ for $1 \mathrm{~h}$ at $37^{\circ} \mathrm{C}$. Cells were then permeabilized to extract soluble tubulin, fixed, and stained for PECAM or VE-cadherin and microtubules using Alexa Fluor 546-conjugated mAbs against PECAM or VEcadherin (red), and for microtubules using anti- $\alpha$-tubulin antibody followed by Alexa Fluor 488-conjugated secondary antibody (green). Note that this prefixation permeabilization sometimes removes small portions of membrane, making cell borders appear discontinuous in both control and DCN-treated cells (however, see Fig. S1). Bar, $10 \mu \mathrm{m}$. (B) HUVEC monolayers were treated with DCN or DMSO (Contr) as in A, incubated with fluorescently labeled nonspecific antibody (NS) or anti-PECAM mAb, and nonenzymatically resuspended and analyzed by flow cytometry. DCN treatment did not affect the levels of surface PECAM at the endothelial borders.

over borders between endothelial cells on which the recycling LBRC membrane appeared as a relatively straight line. However, sometimes monocytes on DCN-treated monolayers appeared to nestle in the junction, and recycled LBRC membrane formed small rings at the interface, presumably where homophilic PECAM-PECAM interactions were taking place. For the purposes of quantitation, we drew our sampling line through the maximum points of intensity along these rings (note that this would bias us against finding an inhibition of LRBC recycling in DCN-treated cells). Even so, enrichment of recycled membrane increased 12-fold in control cells compared with only 2 -fold in these selected DCNtreated cells (Fig. 3 D). These quantitative results are representative of the qualitative enrichment observed for hundreds of 

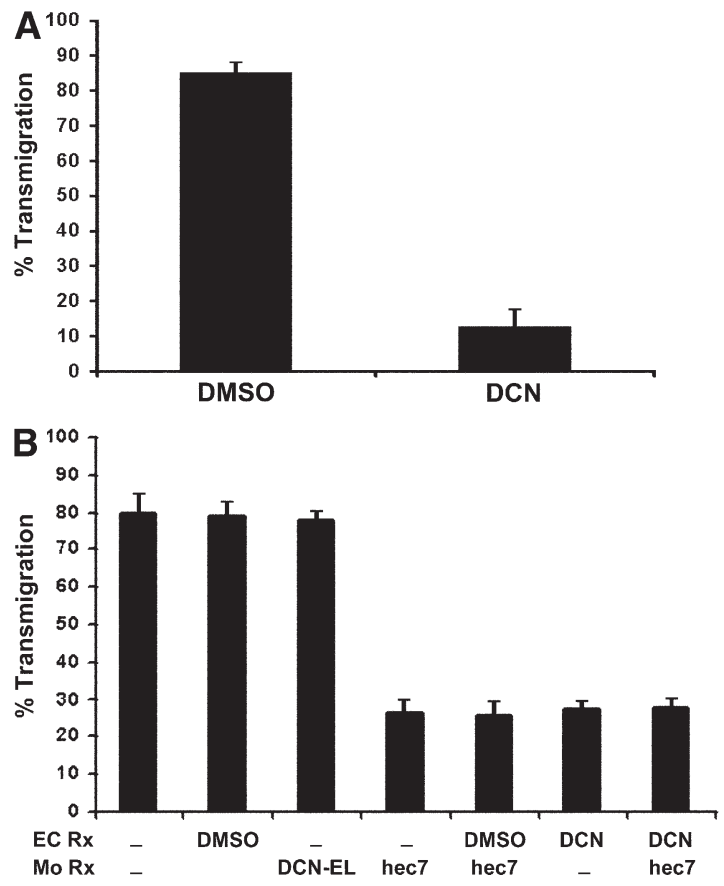

Figure 2. DCN treatment of endothelial cells blocks transmigration of monocytes. (A) Summary of three experiments examining TEM across DCN-treated and control (DMSO carrier-treated) endothelial cells. DCN inhibition of TEM was significant $\left(P=9 \times 10^{-31}\right)$. Data are mean \pm SEM $(n=18$; total independent observations across all experiments involving six monolayers and $>600$ monocytes for each variable in each experiment). (B) Comparison of the block to TEM afforded by DCN and anti-PECAM treatment. These results are combined from three independent experiments in which all variables were examined simultaneously. HUVEC monolayers were treated (EC Rx) for $1 \mathrm{~h}$ with $10 \mu \mathrm{M}$ $\mathrm{DCN}$, the equivalent concentration of DMSO carrier, or left untreated $(-)$, and were then washed extensively before monocytes were added for a typical TEM assay. DCN treatment blocked TEM to the same degree as the blocking anti-PECAM mAb hec7 added to monocytes (Mo Rx). The eluate control (DCN-EL; see Materials and methods) showed that there was no carryover of DCN treatment to affect monocytes. Data shown are mean for all three experiments \pm SEM $(n=6$ per condition for each independent experiment). All inhibitions by hec7 or DCN were significantly different from all controls. $\mathrm{P} \leq 0.00031$ using the Bonferroni correction.

leukocyte-endothelial cell interactions. Equivalent qualitative and quantitative results were seen whether or not the endothelial cells had been activated with TNF- $\alpha$. This block of transmigration, but not of adhesion, and the block in targeted recycling resembled what we previously observed when either leukocytes or endothelial cells were blocked with anti-PECAM antibodies $(5,9)$. This strongly suggested that microtubules were involved in the targeted recycling of the PECAM-containing LBRC, and it demonstrated that targeted recycling of the LBRC and TEM could be controlled by factors other than or in addition to PECAM interactions at the cell surface. However, the same effect might be expected if microtubules were responsible for maintaining the structure and/or distribution of the LBRC.

\section{Depolymerizing microtubules has no effect on the size or location of the LBRC}

To test whether DCN treatment affected the organization or the location of the LBRC, we examined the morphology of the latter in both control and DCN-treated monolayers by immunoelectron microscopy (immuno-EM). We labeled the LBRC in HUVEC monolayers, as described previously (9), and treated the cells with $10 \mu \mathrm{M}$ DCN or DMSO, and processed them for immuno-EM. PECAM-containing vesiclelike structures were seen in proximity to and/or budding from the junctions in control as well as in DCN-treated cells (Fig. 4 A). Quantification of $>100$ cell profiles revealed no difference in the number or the position of these structures in the presence or absence of DCN. These data demonstrate that the structure and the distribution of the PECAM-containing LBRC near the junction are not affected by microtubule depolymerization.

In resting endothelial cells, PECAM-bearing membrane is constantly internalized from the cell border into the LBRC and cycles back evenly from the LBRC along the lateral cell border (9). Although the localization and organization of the LBRC were not altered by DCN treatment, it was possible that defective targeted recycling of this compartment was secondary to perturbation of the constitutive recycling of the LBRC by microtubule disruption. To determine whether constitutive recycling of the LBRC involves microtubules, recycling experiments were performed in control and DCN-treated resting endothelial cell monolayers (in the absence of leukocytes), as described in Materials and methods. DCN treatment had no effect on short-distance, constitutive recycling of membrane (Fig. 4 B) from the LBRC. Based on these findings, we propose that the local constitutive recycling of LBRC membrane at cell borders does not require microtubules, whereas the targeted recycling of membrane from the LBRC to the area of diapedesis is dependent on microtubules.

\section{Blockade of targeted recycling and TEM is reversible}

We tested the effect of another microtubule-destabilizing drug, nocodazole, on TEM. Unlike DCN, nocodazole's effect on microtubules is rapidly reversible (13). When HUVEC monolayers were treated with $2 \mu \mathrm{M}$ nocodazole for $1 \mathrm{~h}$ at $37^{\circ} \mathrm{C}$, their microtubules were depolymerized completely (Fig. S1, available at http://www.jem.org/cgi/content/full/ jem.20072328/DC1). As with DCN, nocodazole did not disrupt the endothelial junctions or the distribution of PECAM at the cell borders. Endothelial cells treated with $2 \mu \mathrm{M}$ nocodazole or DMSO for $1 \mathrm{~h}$ were washed free of drug and used in transmigration assays. We quantified TEM as a function of time as microtubules recovered (Fig. 5). Most monocytes crossed the control endothelial monolayer, reaching plateau levels $(80 \%)$ between 30 and $60 \mathrm{~min}$. In contrast, depolymerization of endothelial microtubules with nocodazole induced a significant block in monocyte TEM that was overcome with time after nocodazole washout. TEM in nocodazole-pretreated cells reached the level of control cells 


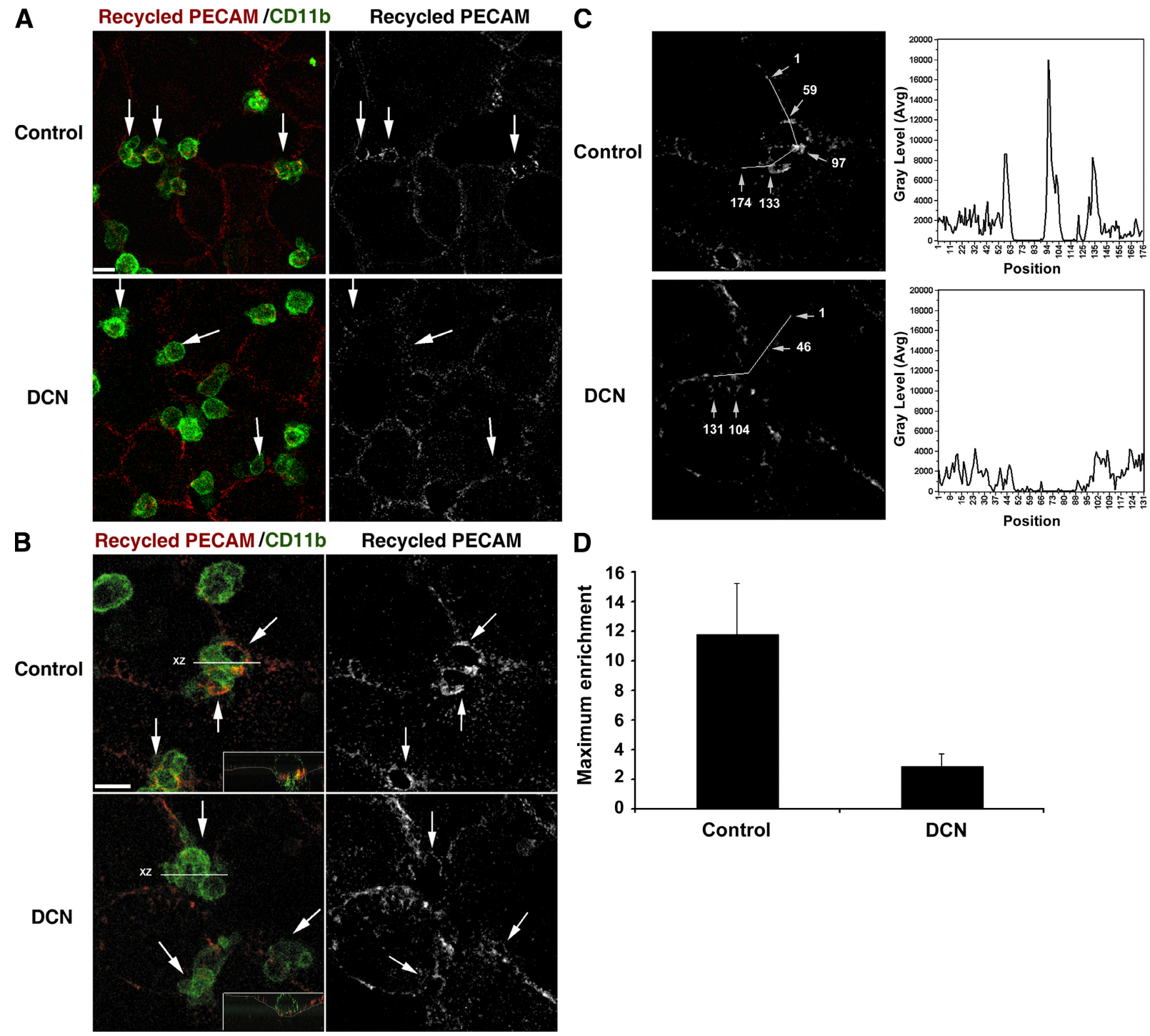

Figure 3. Microtubules are crucial for targeted recycling of PECAM-containing LBRC. HUVEC monolayers were prepared for visualization of targeted recycling of PECAM, treated with DCN or diluent control (see Materials and methods), and incubated with monocytes. Recycling PECAM was visualized with rhodamine-conjugated goat anti-mouse $F\left(a b^{\prime}\right)_{2}$, monocytes with Alexa Fluor 488-labeled anti-CD11b. (A) Representative low-power fields. (B) High-power fields to demonstrate enrichment of recycled PECAM around transmigrating leukocytes. (left) Overlay of the recycled PECAM and CD11b fluorescence channels. (right) Recycled PECAM channel only. Arrows point to representative monocytes. Insets in B show orthogonal views of the monocytes transmigrating in control cells and unable to transmigrate in the DCN-treated cells. The thin white line outlines the endothelial monolayer. Targeted recycling was seen around control monocytes that transmigrated but was not seen around monocytes at the borders between DCN-treated cells, where transmigration was inhibited. Bars, $10 \mu \mathrm{m}$. (C) Illustration of the procedure for quantification of enrichment of membrane recycling from the LBRC (see Materials and methods). Pixel intensity was quantified along the lines shown, drawn to include the endothelial border at the site of leukocyte interaction and away from it. Gray levels are graphed to the right of each picture. The numbers on the fluorescence micrograph (arrows) correspond to positions along the line indicated on the $x$ axis. Two monocytes are transmigrating between approximately positions 60-90 and 105-125 in the control sample, and one monocyte is arrested at the junction between positions 50-85 in the DCN sample. (D) Quantification of maximal enrichment values \pm SD for 14 (control) and 15 (DCN) junctions, as illustrated in $C$ and described in Materials and methods. Enrichment is significantly greater in controls $\left(P=2 \times 10^{-7}\right)$.

by 90 min. Recovery of monocyte TEM after nocodazole washout was concomitant with microtubule repolymerization (Fig. 5, bottom). Recovery of microtubules after nocodazole washout in endothelial cells grown on collagen gels was slower than recovery of the same cells grown on fibronectincoated plastic (10-15 min) and was likely caused by retention of trace amounts of nocodazole in the collagen matrix. However, there was clearly no significant carryover of the drug to the leukocytes, as shown by the eluate control (Fig. 5, top), demonstrating that the effect of nocodazole seen on monocyte TEM is a result of depolymerization of endothelial cell microtubules. 
A

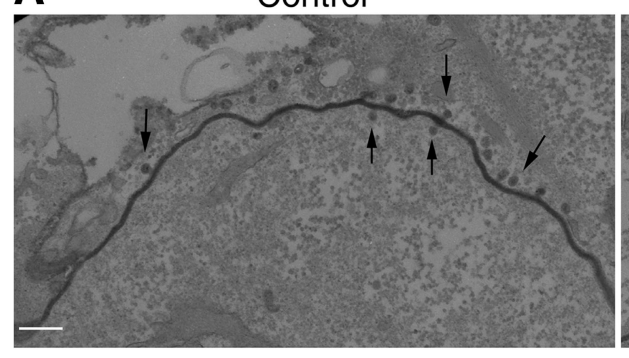

B
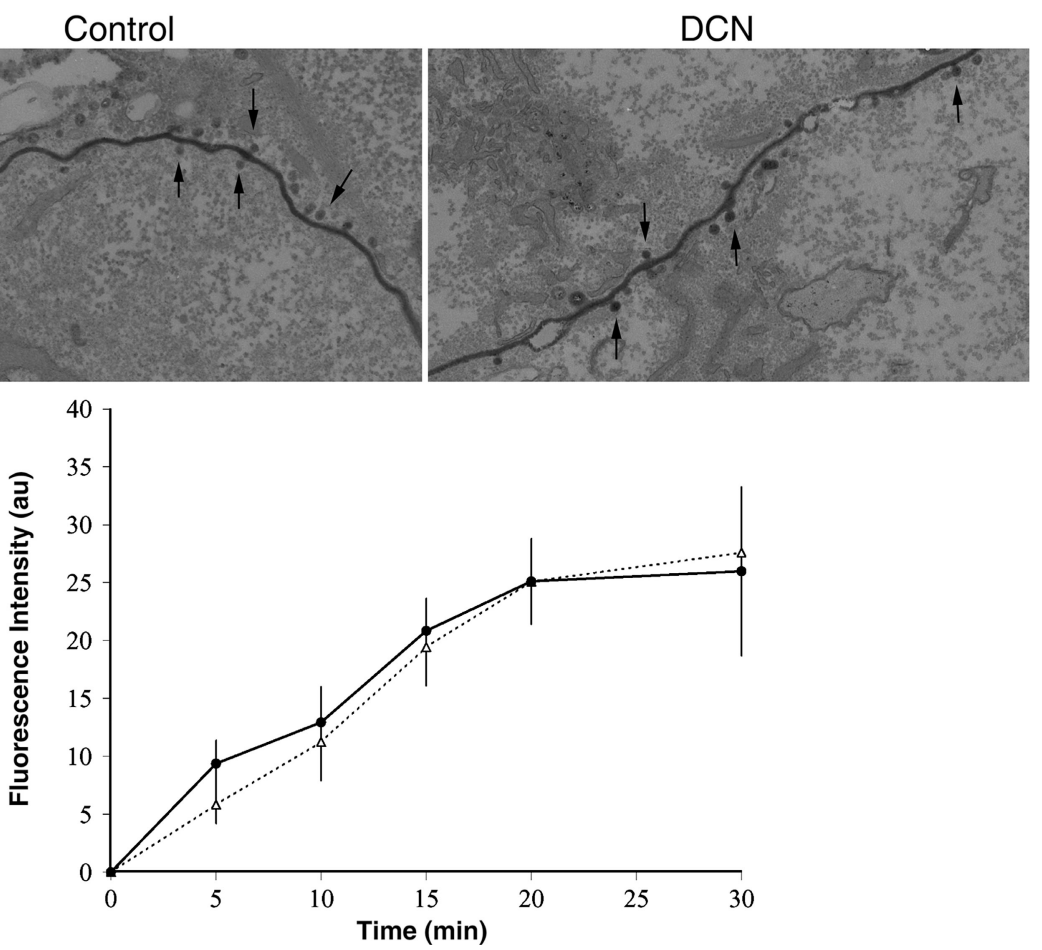

Figure 4. Depolymerization of microtubules does not affect the morphology, localization, or constitutive recycling of the PECAM-containing LBRC. (A) HUVEC monolayers were incubated in the presence of HRP-labeled nonblocking anti-PECAM IgG to saturably label PECAM at the cell borders and in the LBRC before addition of DCN, as described in Materials and methods, and processed for immuno-EM. En face sections of DCN-treated cells show the same morphology and localization of PECAM-containing LBRC (arrows) as control cells. Analysis showed $9.8 \pm 3.4$ (mean \pm SD) vesicles/um in control cells versus $8.9 \pm 2.2$ vesicles/ $\mu \mathrm{m}$ in DCN-treated cells (differences not significant). Bar, $200 \mathrm{~nm}$. (B) Quantitative LBRC recycling experiments were performed on control (continuous line) and DCN-treated HUVEC monolayers (dotted line) in the absence of leukocyte diapedesis, as described in Materials and methods. Pixel intensity at the cell junctions was quantified from digitized images acquired at each time point. Data shown are mean \pm SEM of at least 20 fields acquired from each of two replicates per time point. The experiment was repeated three times with similar results. No significant differences were observed.

\section{Specificity of drug effects on microtubules}

Although DCN and nocodazole are believed to be selective microtubule agonists, no drug is completely specific; microtubule depolymerization with these types of inhibitors may also affect rho GTPases, myosin light chain kinase, and others, causing changes in the distribution of actin and intermediate filaments and redistribution of organelles. We therefore performed a series of experiments to determine whether the block in TEM could be caused by these secondary effects.

The drug taxol, a microtubule agonist, is not known to have these side effects. It stabilizes microtubules and results in accumulation of noncentrosomally organized microtubule bundles in many cell types (14). To determine whether interfering with normal microtubule function in this manner also affected TEM, we incubated endothelial cells for $1 \mathrm{~h}$ with $10 \mu \mathrm{M}$ taxol. This treatment induced bundling of microtubules without altering the endothelial junctions (Fig. S2 A, available at http://www.jem.org/cgi/content/ full/jem.20072328/DC1). The efficacy of taxol treatment was demonstrated by the resistance of microtubules in treated ECs to cold-induced depolymerization (Fig. S2 B). Taxol treatment had no effect on monocyte adhesion to endothelial monolayers but blocked monocyte TEM by 90\% (Fig. S3). Similar to DCN-treated cells, targeted recycling of PECAM was blocked in taxol-treated cells (unpublished data). Furthermore, as with DCN (Fig. 4), taxol treatment did not have any effect on constitutive recycling of the LBRC (Fig. S4).

Of interest, stabilizing actin filaments in endothelial cells with the drug jasplakinolide had a very modest, if any, effect on transmigration (Fig. S3). This suggests that dynamic changes in actin filaments are not involved in targeted recycling of the LBRC. We were unable to effectively depolymerize actin filaments without compromising the integrity of the monolayer, so we were unable to perform the converse experiment.

Microtubule depolymerization has been shown to induce perinuclear clustering of mitochondria in several cell types. This raised the possibility that inhibition of TEM by microtubule-depolymerizing agents might be caused by depravation of a local source of concentrated ATP necessary to support diapedesis. Migrating lymphocytes require polarized mitochondria for chemotaxis (15). However, direct observation of mitochondria in DCN-treated cells showed that perinuclear collapse of mitochondria required overnight drug treatment, 

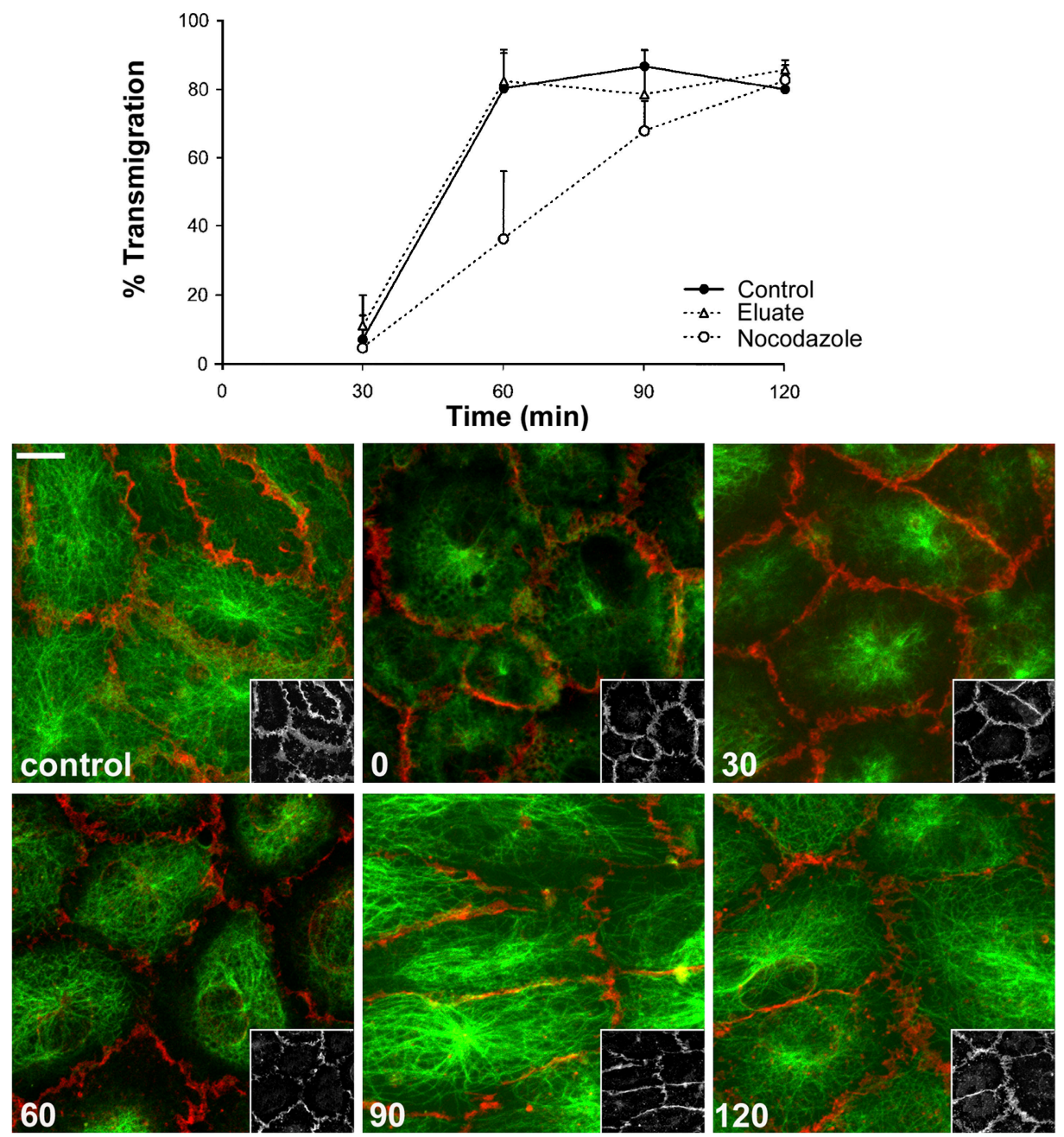

Figure 5. The effect of microtubule-depolymerizing drugs on monocyte TEM is reversible. HUVEC monolayers were incubated in $2 \mu M$ nocodazole or diluent control for $60 \mathrm{~min}$ at $37^{\circ} \mathrm{C}$. After extensive washing, freshly isolated PBMCs were added on the top of both control and nocodazoletreated HUVEC monolayers, and the co-cultures were incubated for different time periods at $37^{\circ} \mathrm{C}$. A replicate set of monocytes was resuspended in conditioned medium from nocodazole-treated HUVEC monolayers (eluate) to control for carryover of nocodazole (see Materials and methods). After washing and fixing the cells, monocyte TEM was quantified (top), and microtubule morphology (bottom) was examined at each time point. Data are mean \pm SEM for six to eight random fields on each of five replicates per time point. Nocodazole treatment significantly reduced transmigration at 60 and 90 min $(P=0.0021$ and 0.0056 , respectively) but not at $120 \mathrm{~min}(P=0.63)$. The experiment was repeated three times with similar results. The bottom panels show microtubules stained in green and total PECAM stained in red. These monolayers were not extracted to remove soluble tubulin, which stains in an amorphous and partially reticular pattern in the cytoplasm at time zero, as it is dispersed between other organelles. Note that monolayers remain intact, as assessed by maintenance of peripheral PECAM staining, and there is progressive recovery and centrifugal growth of microtubules as a function of time. Because peripheral microtubules sometimes obscure PECAM staining in the merged images, insets show the PECAM channel of the same field at a lower magnification. Numbers show the time in minutes after nocodazole washout. Bar, $10 \mu \mathrm{m}$.

whereas mitochondria remained at the cell periphery during the 1-h treatment we used to block targeted recycling and TEM (Fig. S5 A, available at http://www.jem.org/cgi/content/ full/jem.20072328/DC1). Additionally, mitochondria remain peripherally distributed in the taxol-treated cells, which do not support TEM. Thus, changes in mitochondrial distribution are not the reason for nocodazole or DCN-induced inhibition of TEM and targeted recycling.

Similarly, disrupting microtubules can cause centripetal redistribution of the endoplasmic reticulum, which may affect calcium signaling (16) that is required for TEM (17). However, treatment of HUVEC monolayers with DCN under the conditions used for our TEM experiments had no effect on the distribution of endoplasmic reticulum (Fig. S5 B).

Furthermore, we directly tested the effect of this DCN treatment on endothelial calcium signaling in response to $10 \mu \mathrm{M}$ histamine, which produces a similar increase in cytosolic free calcium $\left(\uparrow\left[\mathrm{Ca}^{+2}\right]_{\mathrm{i}}\right)$ to that of leukocyte-endothelial cell interactions (17). There was no significant difference in the responses of control and DCN-treated endothelial 
cells (Fig. S5 D). In all of these experiments, staining for $\alpha$-tubulin demonstrated that the DCN treatment effectively depolymerized microtubules.

\section{Microtubules are also required for PECAM-independent transmigration}

Inhibition of PECAM function and of microtubule integrity resulted in the same phenotype: leukocytes blocked at the apical surface of the endothelial cell border without targeted recycling from the LBRC. However, we were interested to know whether this was a general phenomenon, and in particular, whether PECAM-independent transmigration also depended on intact microtubules and trafficking from the LBRC. Polyclonally activated human T cells transmigrate our cytokine-activated HUVEC monolayers in reasonable numbers. Unlike transmigration of monocytes and neutrophils, activated lymphocyte migration cannot be significantly inhibited by anti-PECAM blocking mAbs (unpublished data) $(18,19)$. We examined transmigration of monocytes and lymphocytes across TNF- $\alpha$-activated HUVEC monolayers treated with DCN to depolymerize microtubules. Transmigration of both cell types was markedly reduced on the DCN-treated monolayers (Fig. 6 A). As usual, the eluate control demonstrated that the effect of DCN was only on the endothelial cells. Moreover, targeted recycling from the LBRC was seen around T cells transmigrating control monolayers but not around those stuck at the junctions of DCN-treated monolayers (Fig. 6 B). Thus, leukocyte transmigration requires intact endothelial microtubules regardless of whether it requires PECAM.

\section{Targeted recycling and TEM require kinesin family molecular motors}

The preceding data support the idea that intact microtubules are essential for leukocyte TEM by bringing the LBRC to the site of diapedesis. Because the kinesin family of microtubule motors can transport vesicles toward microtubule plus ends (toward the periphery of endothelial cells; for review see reference 11), we next explored whether kinesin-mediated transport was involved in the targeted trafficking of the LBRC to the site of TEM. Of the 41 known human kinesin family members, 30 are expressed at the mRNA or protein level in HUVECs (Table S1, available at http://www.jem .org/cgi/content/full/jem.20072328/DC1). This rendered attempts to reduce their levels singly and in combination by methods such as RNA interference impractical. Instead, we chose to inhibit kinesin activity directly by microinjection of HUVEC monolayers with a function-blocking antibody, known as HD, that was raised against the highly conserved motor domain of Drosophila kinesin heavy chain (20). Immunoblot analysis and functional studies suggest that HD recognizes several kinesin family members in addition to KIF5 (conventional kinesin) (21) and, thus, could likely inhibit multiple kinesin family members in HUVECs.

The organization of mitochondria is dependent on microtubules and kinesins (22-24). In addition, microinjected HD

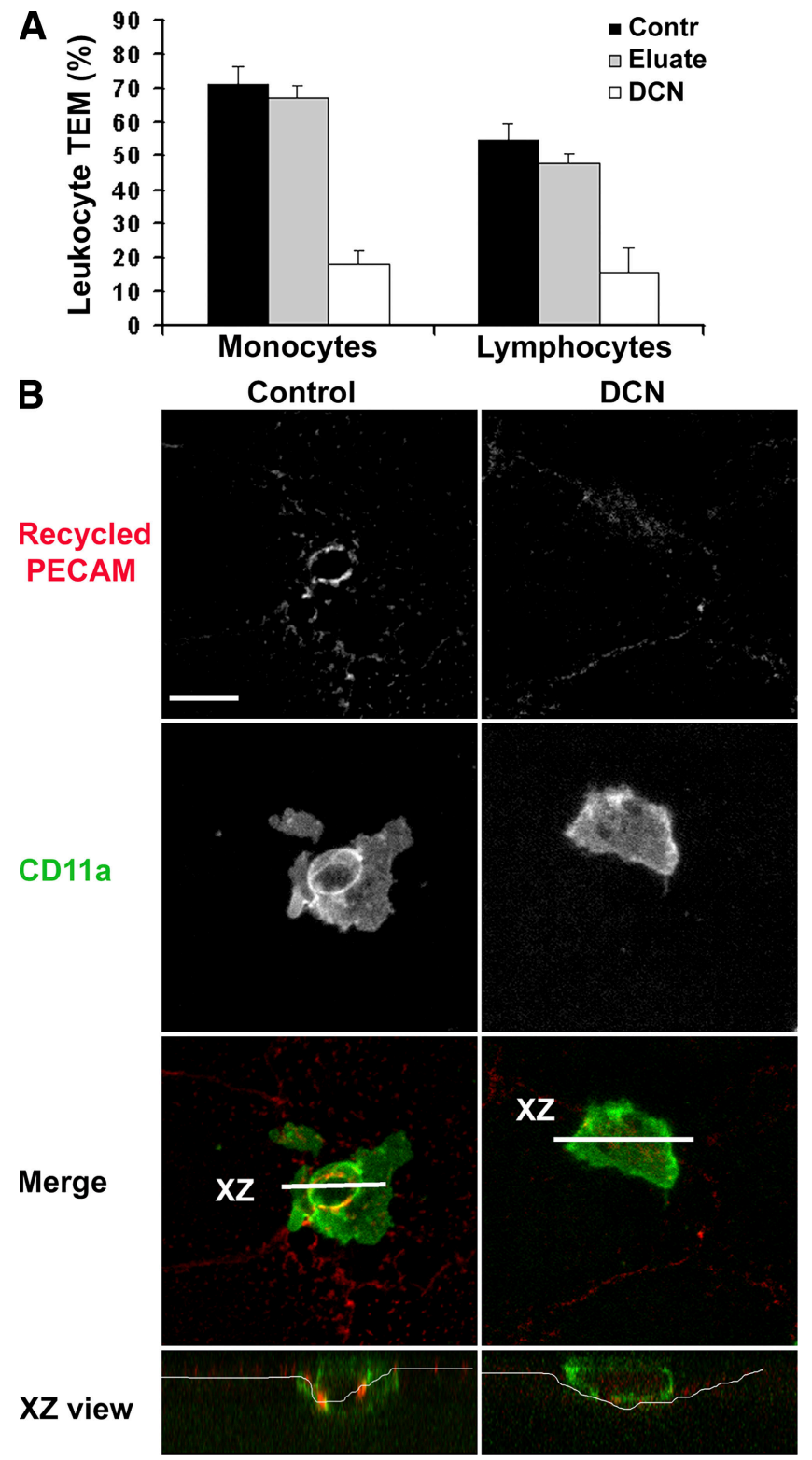

Figure 6. Intact microtubules are required for transmigration of lymphocytes. (A) HUVEC monolayers were activated with TNF- $\alpha$, as described in Materials and methods, and treated or not with DCN before the addition of monocytes or polyclonally activated cultured T cells. Transmigration was allowed to proceed for $1 \mathrm{~h}$. One set of leukocytes was resuspended in conditioned medium washed out of DCN-treated HUVECS (eluate) as a control for DCN carryover, and then added to the endothelial cells. Data shown are mean \pm SD for eight observations per sample. Transmigration across DCN-treated monolayers is significantly inhibited compared with controls for both monocytes and lymphocytes $\left(P=1.2 \times 10^{-12}\right.$ and $5.5 \times 10^{-4}$, respectively, using the Bonferroni correction). (B) Representative images from a typical experiment demonstrating that targeted recycling of $\angle B R C$ membrane is inhibited in DCN-treated endothelial monolayers. Recycled PECAM was visualized as in the previous figures; lymphocytes were visualized with fluorescent CD11a (green). Merged images show enrichment of recycling LBRC membrane around transmigrating lymphocytes on control but not DCN-treated endothelium (see legend to Fig. 3). 
IgG was previously shown to alter the distribution of mitochondria (23). Thus, as a test of HD efficacy in endothelial cells, we first examined whether it inhibited centrifugal redistribution of mitochondria after washout of microtubule antagonists in endothelial cells. We first induced perinuclear accumulation of microtubules with nocodazole, and then injected HD or control rabbit IgG, washed out the nocodazole, and monitored the redistribution of microtubules and mitochondria. In both sets of cells, microtubules rapidly repoly- merized after washout of nocodazole. In cells injected with HD IgG, mitochondria remained centrally located, whereas mitochondria in cells injected with the same concentration of normal rabbit IgG moved out to the cell periphery and within $1 \mathrm{~h}$ resembled untreated cells (Fig. S6). Thus, injected $\mathrm{HD}$ is able to block a known kinesin function in endothelial cells. Note, however, that although HD IgG prevented the centrifugal redistribution of previously collapsed mitochondria, there was no significant effect on redistribution of
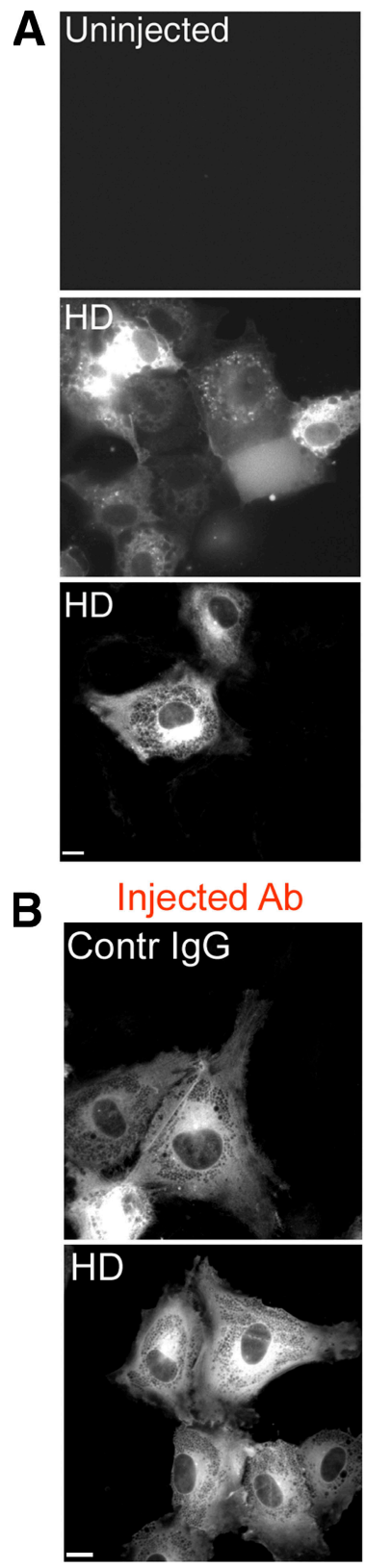
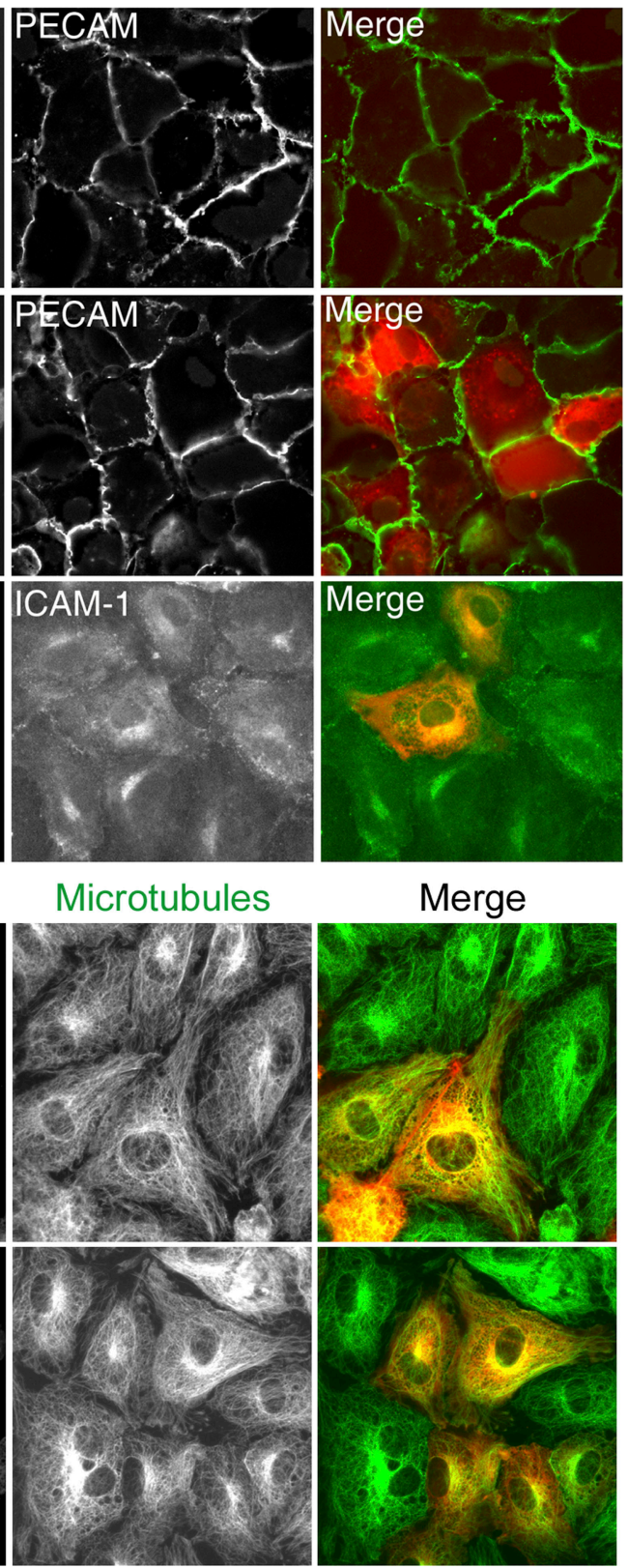

Figure 7. Blocking kinesin motor domain has no effect on PECAM or ICAM-1 expression, nor on distribution of microtubules. (A) TNF- $\alpha-$ stimulated HUVECs injected with HD IgG were incubated for $6 \mathrm{~h}$ and stained for PECAM or ICAM-1 expression. Alexa Fluor 546-labeled goat anti-rabbit $\lg \mathrm{G}$ was used to visualize injected cells. No difference in expression was seen between injected and uninjected cells. Bar, $10 \mu \mathrm{m}$. (B) HUVECs treated and microinjected with HD or control lgG as in A were washed and fixed in methanol and stained to visualize microtubules (green) or to visualize injected antibody (red). Bar, $10 \mu \mathrm{m}$. 
mitochondria over the time course of our experiments when injected into untreated cells (not depicted), similar to the lack of effect of DCN on mitochondrial distribution (Fig. S5 A). Furthermore, microinjection of control or HD IgG had no effect on the distribution of intermediate filaments, as assessed by vimentin staining (Fig. S5 C).

Important for our next series of experiments, microinjection of endothelial cells with HD antibody had no effect on the expression or distribution of intercellular adhesion molecule (ICAM) 1 or PECAM (Fig. 7 A), or of microtubules (Fig. 7 B). To demonstrate this, Fig. 7 intentionally shows fields in which some cells are uninjected and in which injected cells contain different amounts of antibody for direct comparison. We performed TEM experiments on monolayers in which large patches of adjacent endothelial cells were injected with HD or control antibodies. After the assay, monolayers were fixed and stained to identify the injected endothelial cells and examined by confocal microscopy to assess adhesion and transmigration. There was no significant difference in the adhesion of monocytes to endothelial cells injected with HD IgG compared with control antibody (Fig. 8 A). However, TEM was decreased by $>80 \%$ across cells injected with the anti-kinesin motor domain antibody compared with cells injected with control antibody (Fig. 8 B).

When we examined targeted recycling of the LBRC in these experiments, we found that the compartment recycled to surround leukocytes transmigrating across uninjected or control IgG-injected endothelial cells, as expected (Fig. 9). However, although leukocytes could adhere normally to the surfaces of endothelial cells injected with HD IgG (Fig. 8 A) and crawl to the intercellular borders, targeted recycling to the vicinity of leukocytes adherent to these cells was not observed (Fig. 9). Leukocytes remained adherent to the apical surface of the endothelium straddling the junction, whereas membrane from the LBRC recycled evenly along the borders without enrichment at points of leukocyte-endothelial cell contact. These co-cultures resembled those in which microtubules were depolymerized with DCN (Fig. 3) or blocked with anti-PECAM mAb (9). However, in these cells microtubules were intact and PECAM was unperturbed. We conclude that targeted recycling from the LBRC to the site of diapedesis is required for TEM, and that this requires both intact microtubules and functional kinesin, presumably for kinesin-mediated movement of LBRC membrane along intact microtubules.

\section{DISCUSSION}

Our knowledge of the molecules involved in diapedesis has increased dramatically in the past $15 \mathrm{yr}$. PECAM was the first molecule demonstrated to play a unique role in diapedesis unrelated to leukocyte-endothelial cell adhesion. Homophilic interaction between PECAM on the leukocyte and PECAM concentrated at the endothelial cell border is critical for diapedesis of adherent leukocytes to proceed (5). A similar homophilic interaction between leukocyte CD99 and CD99 concentrated at the endothelial border controls a later step in
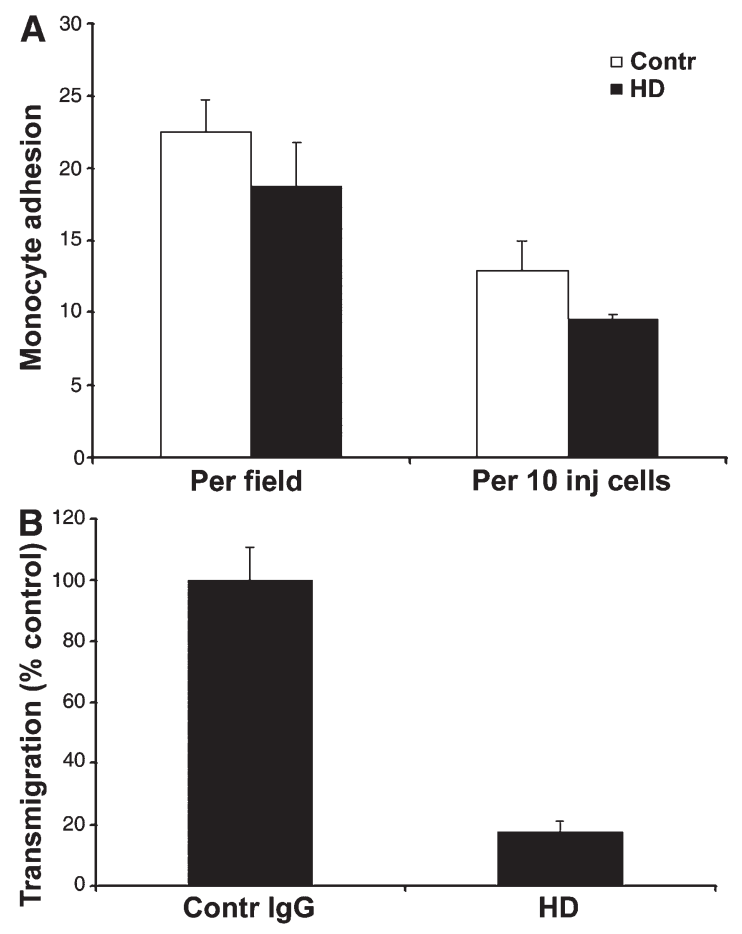

Figure 8. Blocking kinesin motor domain selectively blocks TEM. (A) HUVECs activated for $4 \mathrm{~h}$ with TNF- $\alpha$ and injected with HD or control $\lg \mathrm{G}$ were incubated with monocytes. The total number of monocytes adherent to endothelial cells injected with control or anti-kinesin antibody was quantified from observations of hundreds of leukocytes interacting with endothelial cells in at least 16 random fields from three replicate dishes. Data are expressed as adhesions per field (including uninjected and injected cells) or as adhesion to injected (inj) cells only. Data are mean \pm SEM. The small apparent difference in adhesion is not statistically significant. (B) Monocytes in the experiment shown in A were further analyzed for TEM. The percentage of adherent monocytes that migrated across the endothelial monolayer is shown as the mean \pm SEM for the same number of fields and replicates. Anti-kinesin antibody blocked transmigration by $>80 \%(P=0.00386)$. These results are representative of three independent experiments. 602 monocytes were counted for control monolayers and 271 were counted for HD-injected monolayers, interacting with 305 HUVECS injected with control lgG and 163 HUVECs injected with HD.

transmigration and is critical for leukocytes to complete diapedesis (12). Several other molecules of the endothelial junction, including ICAM-2, junctional adhesion molecule A, endothelial cell-selective adhesion molecule, and poliovirus receptor, have been implicated in diapedesis (25-28). As with PECAM and CD99, evidence for the involvement of these molecules comes from blocking experiments in vitro and antibody blocking or genetic ablation experiments in vivo. These studies demonstrate a necessary role for these molecules in diapedesis. However, the mechanisms through which these molecules regulate diapedesis are still largely unknown.

Insights into the mechanism began to unfold when we reported that $\sim 30 \%$ of the total endothelial cell PECAM was present in the LBRC, a reticulum of vesicle-like structures connected to each other and to the surface of the cell at the intercellular borders (9). Although this membrane recycled 

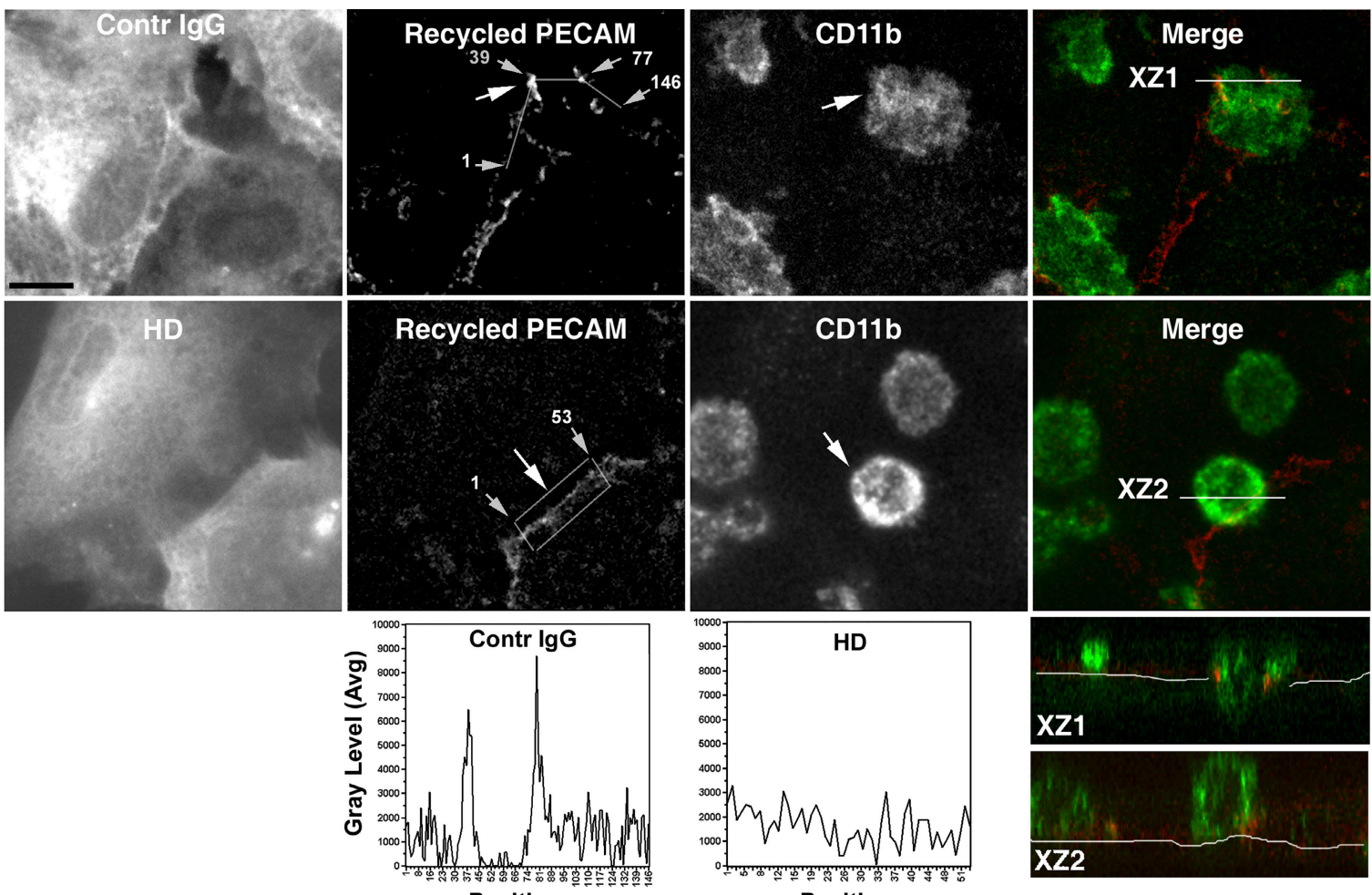

Position

Position

Figure 9. Blocking kinesin motor domain blocks targeted recycling of membrane from the LBRC. Cytokine-activated endothelial cells were microinjected with nonimmune rabbit lgG (control) or HD IgG and used in targeted recycling experiments, as described in Materials and methods. Each of the top two rows shows the same representative field of z-series projections of confocal images. Individual panels show monochrome images of the location of injected antibody (far red, displayed as white), recycled PECAM (red), and monocytes stained with anti-CD11b (green). The far right panels show merged images of recycled PECAM and CD11b; the bottom panels show an orthogonal (X-Z) projection through the stacks along the line indicated in white. Note that although transmigration and targeted recycling occurred normally in cells injected with nonimmune IgG (XZ1), transmigration and targeted recycling of membrane from the LBRC were blocked in cells injected with the HD antibody (XZ2), and membrane recycled evenly along the intercellular border. The fluorescence intensity of recycled PECAM along the line drawn clockwise around the transmigrating monocyte (indicated by a large arrow) for control IgG and along a line drawn through the center of the rectangle for HD (not depicted, so as not to obscure the original data) are graphed below the micrographs. Numbers on micrographs (small arrows) correspond to the position along the measured line, as in Fig. 3. Bar, 10 $\mu$ m.

rapidly and evenly between the junctional surface and the LBRC in resting endothelial cells, during diapedesis, this membrane trafficking was redirected to the site on the endothelial cell border where the leukocyte was in the process of transmigrating. Although we see a clear enrichment of fluorescence representing recycling membrane trafficking to the transmigration zone, we have not seen the three dimensional "docking sites" or "transmigratory cups" described by Barreiro et al. (29) and Carman et al. (30). Such structures were also not seen by other investigators (31) studying diapedesis in vitro. Differences in our results are likely caused by differences in the experimental systems used. Homophilic interaction between leukocyte and endothelial PECAM triggered this targeted recycling (9), and TEM was not observed in the absence of targeted recycling. However, it was unclear whether this recycling by itself was required for TEM or whether this phenomenon was independent of PECAM.

This raised the question of how membrane from the LBRC is targeted to the zone of leukocyte diapedesis. The LBRC appears to be a unique organelle, organized differently from known endocytic vesicular systems and not even composed of true vesicles, because the compartment is never sealed off completely from the junctional surface (9). Thus, it was not clear how trafficking of this membrane would occur. We report in this paper that targeted recycling of membrane from the LBRC to the site of diapedesis requires intact and normally functioning microtubules and active kinesin motors. Although we are unable to visualize this directly, the most logical mechanism would be that LBRC membrane is moved along microtubules to the site of diapedesis by kinesin. Disrupting microtubules (Figs. 2, 3, and 5; and Fig. S3) or interfering with kinesins (Figs. 8 and 9) blocked diapedesis without affecting the local, constitutive recycling of PECAM from the LBRC or the adhesion of leukocytes. The phenotype of the arrested cells resembled that seen when homophilic interactions of leukocyte and endothelial cell PECAM were inhibited using blocking antibodies (9). However, in the present study, no PECAM function-blocking reagents were necessary to disrupt targeted recycling and diapedesis. The fact that depolymerization of endothelial microtubules, inhibition of 
kinesin motor function, and blockade of PECAM function by antibody inhibited TEM to the same extent (Figs. 2 and 8) and were not additive (Fig. 2) suggests that these perturbations block TEM along the same functional pathway. For the same reason, we hypothesize that blockade of PECAM function by antibodies and inhibition of kinesin motors by HD would not be additive, but we have not tested this. Although we cannot rule out additional roles of microtubules and kinesin molecular motors in TEM, we propose that homophilic engagement of leukocyte PECAM with endothelial PECAM at the apical surface of the endothelial cell border sends a signal that triggers recruitment of membrane from the LBRC to the site of diapedesis, and that this targeted recruitment requires kinesin-mediated, microtubule-based transport. Of course, other molecules at the endothelial border and/or in the LBRC may participate in this signaling process downstream of PECAM. Similarly, even though the ability of endothelial cells to flux cytosolic-free calcium was not affected by dissolution of microtubules under our experimental conditions, this does not mean that calcium signaling may not play a role in the targeted recycling of LBRC membrane. Our data also demonstrate that targeted membrane recycling from the LBRC is itself required for diapedesis, because inhibiting targeted recycling independently of PECAM blocked diapedesis effectively.

TEM was rarely observed across endothelial cells injected with the function-blocking anti-kinesin antibody HD. In fact, in those cultures, monocytes appeared to transmigrate in high numbers across uninjected endothelial cells at the borders of the injected patches (unpublished data). Adherent leukocytes that cannot transmigrate because PECAM is blocked crawl along the junctions of endothelial monolayers, from endothelial cell to endothelial cell, as if searching for a place to diapedese (32). The extra monocytes that did transmigrate across the uninjected endothelial cells adjacent to the HD-injected ones likely represent leukocytes that were unable to transmigrate the HD-injected endothelial cells and crawled across the monolayer until they came to uninjected cells. This behavior was not seen in cells injected with control antibody.

The fact that stabilization of microtubules with taxol also selectively blocked TEM (Figs. S2-S4) suggests that dynamic rather than static microtubules may be necessary for targeted recycling from the LBRC. Given the technical limitations of our experimental system, we are unable to directly visualize recycling LBRC membrane moving along microtubules in living cells. Thus, we cannot completely rule out the possibility that microtubules and kinesin motors serve as an anchoring platform for targeted recycling rather than acting to actively transport vesicles. However, the fact that taxol, which would not be expected to alter such a scaffolding structure, also blocked targeted recycling and transmigration would argue against this. Neither taxol nor the microtubule-depolymerizing drugs had a detectable effect on the structure of the LBRC or kinetics of constitutive recycling. We hypothesize that constitutive recycling of this compartment may be governed by myosin motors on actin filaments, but the regulation and purpose of constitutive recycling of LBRC membrane are subjects for future study.
We studied TEM in the absence of fluid shear in our assays. Unfortunately, performing these assays under flow is not technically feasible at this time. Endothelial cells grown on collagen gels cannot be microinjected or used in available perfusion chambers. Endothelial cells grown on glass coverslips (as they would be for standard flow chamber systems) do not make a physiological basement membrane, as they do on collagen gels, and thus, do not adhere to the substrate as tightly. When treated with DCN, they lift off in large patches when subjected to shear flow. However, everything that we have published using our static transmigration system in vitro has been borne out in vivo. This includes the role of Mac-1 in locomotion $(32,33)$, roles for $\operatorname{PECAM}(5,7,8)$ and CD99 $(12,34,35)$ in transmigration (but not adhesion), and the effects of prolonged (days) culture of transmigrated monocytes in our system on their differentiation into immature dendritic cells and monocytes $(36,37)$. Furthermore, studies on the transmigration of myeloid cells show that flow may increase the rate of transmigration quantitatively but does not change any aspects qualitatively $(38,39)$.

Vesicles similar to the LBRC exist in vivo (unpublished data), but it is not possible to study targeted recycling in vivo with our current markers. We hope that isolation of the LBRC compartment by subcellular fractionation will lead to the identification of unique markers for the LBRC that are suitable for use in vivo.

Although it is clear that targeted recycling from the LBRC is critical for diapedesis, the function served by this process is not known. Targeted recycling would be expected to increase the membrane surface area at the cell border, thus facilitating leukocyte passage without the need for endothelial retraction. Moreover, although PECAM, CD99, and VEcadherin are all internalized from the endothelial cell border (9), only PECAM and CD99 appear to be part of the LBRC (unpublished data). VE-cadherin along the cell border temporarily moves out of the way as leukocytes transmigrate $(40,41)$. One scenario to explain these observations is that LBRC membrane (containing PECAM and CD99, but not VE-cadherin) targeted to surround the leukocyte physically displaces the junctional membrane rich in VE-cadherin, thus creating a temporary zone for transmigration without disrupting complex junctional structures. Alternatively, VE-cadherin may be internalized into a separate vacuolar compartment.

Homophilic interaction of PECAM and CD99 on leukocytes with the same molecules at the endothelial cell border is necessary for transmigration. However, at the endothelial cell border, these molecules are already engaged in homophilic interactions with the same molecules on the apposing endothelial cell. Another potential function of LBRC trafficking could be to bring unligated PECAM, CD99, and/or other components of the LBRC into contact with their ligands on leukocytes to initiate and propagate the signals for further recruitment of this membrane farther along the junction as the leukocyte moves through. Alternatively, or additionally, the accretion of free PECAM-bearing (or other components of the LBRC) membrane in the vicinity of the leukocyte 
might create a haptotactic gradient for the leukocyte to follow through the junction (9).

The significance of our observations extends beyond PECAM. Our data show that targeted recycling of membrane from the LBRC is required for transmigration. Polyclonally activated lymphocytes, whose TEM cannot be blocked by anti-PECAM antibody, still require the LBRC for transmigration, because disruption of microtubules blocked TEM to the same extent to which it blocked TEM of PECAM-sensitive monocytes (Fig. 6). Although many transmigration events in vivo require PECAM (1), there are clearly inflammatory situations that are PECAM independent $(25,42)$. Many of the PECAM-independent inflammatory conditions involve leukocytes that have been directly stimulated by application of exogenous chemokines. We hypothesize that in these situations other adhesion molecule interactions and/or signals from leukocyte to endothelium serve to recruit membrane from the LBRC to the zone of diapedesis, substituting for the role otherwise played by PECAM.

To our knowledge, this is the first demonstration of a role for endothelial microtubules and microtubule motors in diapedesis. The next challenges will be to study microtubule dynamics in endothelial cells during TEM, to determine which of the many kinesins expressed by endothelium are responsible for targeted recycling, and to determine whether they would make reasonable antiinflammatory therapeutic targets.

\section{MATERIALS AND METHODS}

Reagents. hec1 (anti-VE-cadherin), P1.1 (anti-CD31), hec7 (anti-CD31), and OKM-1 (anti-CD11b), as previously described (9), were fluorescently labeled with Alexa Fluor 488 or Alexa Fluor 546 according to the manufacturer's instruction (Invitrogen). Unlabeled and rhodamine-conjugated goat anti-mouse $\mathrm{F}\left(\mathrm{ab}^{\prime}\right)_{2}$ antibodies used for PECAM recycling experiments purchased from Jackson ImmunoResearch Laboratories. Anti- $\alpha$-tubulin antibody was purchased from Accurate Chemical. Goat anti-mouse secondary antibodies conjugated to either Alexa Fluor 546 or Alexa Fluor 488 were purchased from Invitrogen. DCN was purchased from EMD. Nocodazole, taxol, and diaminobenzidine were purchased from Sigma-Aldrich.

Cell culture. HUVECs were isolated from fresh umbilical cords, as previously described (3), and grown in medium 199 (M199; Invitrogen) supplemented with $20 \%$ adult human serum and $100 \mathrm{U} / \mathrm{ml}$ penicillin-streptomycin at $37^{\circ} \mathrm{C}$ in a humidified atmosphere of $5 \% \mathrm{CO}_{2}$. Experiments were done routinely with cells at passage two plated on thick hydrated type I collagen gels in 96-well culture plates, or on glass coverslip dishes (Carolina Biological Supply) coated with $5 \mu \mathrm{g} / \mathrm{ml}$ fibronectin (Sigma-Aldrich). In some experiments, endothelial cells were activated by incubation with $10 \mathrm{ng} / \mathrm{ml}$ TNF- $\alpha$ for $\geq 4$ h before the addition of leukocytes.

PBMCs were isolated from healthy volunteers by density gradient centrifugation in Ficoll-Paque, as previously described (43). In some experiments, $\mathrm{T}$ cells were isolated from this mixture by adhesion of monocytes to a 100-mm tissue culture plate. Nonadherent T cells were removed and cultured in RPMI 1640 (Invitrogen) supplemented with 10\% fetal bovine serum, $100 \mathrm{U} / \mathrm{ml}$ penicillin-streptomycin, and $1 \mu \mathrm{g} / \mathrm{ml}$ PHA for $3 \mathrm{~d}$, followed by culture in $20 \mathrm{ng} / \mathrm{ml} \mathrm{IL}-2$ for 3-6 d (30). All experimental protocols were approved by the Weill Cornell Medical College Institutional Review Board.

Immunofluorescence. To visualize the effects of treatments on microtubules, HUVEC monolayers were incubated for $1 \mathrm{~h}$ at $37^{\circ} \mathrm{C}$ in $\mathrm{M} 199$ containing 0.1 human serum albumin plus $10 \mu \mathrm{M}$ DCN, $10 \mu \mathrm{M}$ taxol, $2 \mu \mathrm{M}$ nocodazole, or DMSO (control) at the concentration used to dissolve each drug (always $<0.05 \%$ ). Cells were then rinsed in prewarmed divalent cation-containing PBS and fixed for $1 \mathrm{~min}$ in cold $\left(-80^{\circ} \mathrm{C}\right)$ methanol. In some experiments, cell monolayers were permeabilized by exposure to saponin before fixation to allow extraction of soluble tubulin. In these experiments, cells were washed in PEM buffer (100 mM Pipes, $5 \mathrm{mM}$ EGTA, $5 \mathrm{mM}$ $\mathrm{MgCl}_{2}, \mathrm{pH}$ 8.6) and exposed for $45 \mathrm{~s}$ to $0.3 \mathrm{mg} / \mathrm{ml}$ saponin in PEM, followed by washes in PEM before methanol fixation. Microtubules were visualized by indirect immunostaining using a mouse anti- $\alpha$-tubulin antibody detected by an Alexa Fluor 546-rabbit anti-mouse antibody. In some experiments, mitochondria were stained using Mitotracker (Invitrogen) by incubation at $1 \mu \mathrm{M}$ for $30 \mathrm{~min}$ at $37^{\circ} \mathrm{C}$. Intermediate filaments were labeled with 56B5 mouse antivimentin IgM (a gift from Ron Liem, Columbia University, New York, NY), followed by FITC-anti-Mouse IgM. The endoplasmic reticulum was imaged using ER tracker (Invitrogen). Junctions were visualized by directly conjugated Alexa Fluor 488-hec1 for VE-cadherin or Alexa Fluor 488-P1.1 for PECAM.

To quantitatively analyze the cell-surface expression of PECAM in the presence and absence of microtubule depolymerization, HUVEC monolayers treated with DCN or DMSO were incubated with Alexa Fluor 546conjugated anti-PECAM mAb (clone P1.1) or nonspecific control antibody conjugated to the same fluorophore for $1 \mathrm{~h}$ at $37^{\circ} \mathrm{C}$ to saturate the cell border and LBRC compartments, and were then washed and resuspended by incubation in $10 \mu \mathrm{M}$ EDTA in HBSS at $37^{\circ} \mathrm{C}$. Reuspended cells were washed in HBSS, fixed in paraformaldehyde, and analyzed on a flow cytometer (FACSCalibur) using CellQuest software (both from BD Biosciences).

Permeability assay. HUVEC monolayers on collagen gels were treated with $10 \mu \mathrm{M}$ DCN or DMSO (control), as for the transmigration assay (see the following section), and incubated with Alexa Fluor 546-conjugated albumin (Invitrogen) at $20 \mu \mathrm{g} / \mathrm{ml}$ in culture medium for $1 \mathrm{~h}$ at $37^{\circ} \mathrm{C}$. Monolayers were washed, and fluorescence was read in a fluorescence plate reader (CytoFluor 2350; PerSeptive Biosystems).

Quantitative TEM assay. This assay was performed and quantified as previously described (5). In brief, PBMCs from healthy volunteers were purified on a Ficoll density gradient. They were then washed in HBSS plus $0.1 \%$ human serum albumin and resuspended in M199 plus $0.1 \%$ human serum albumin. $2 \times 10^{5}$ cells were added to each replicate well of confluent monolayers of HUVECs grown on hydrated collagen gels and incubated for $1 \mathrm{~h}$ at $37^{\circ} \mathrm{C}$ in a $\mathrm{CO}_{2}$ incubator. Transmigration assays were performed using both "resting" and TNF- $\alpha$-activated HUVECs. In some experiments, the assay was performed using polyclonally stimulated human T cells on TNF- $\alpha$-activated HUVECs with $200 \mathrm{ng} / \mathrm{ml}$ of stromal cell-derived factor 1 added to the collagen $20 \mathrm{~min}$ before addition of the lymphocytes.

To assess the role of microtubules on monocyte TEM, HUVEC monolayers were pretreated with DMSO (control), $10 \mu \mathrm{M}$ DCN, $2 \mu \mathrm{M}$ nocodazole, or $10 \mu \mathrm{M}$ taxol at $37^{\circ} \mathrm{C}$ for $1 \mathrm{~h}$. They were subsequently washed extensively with cold divalent cation-containing PBS plus $0.1 \%$ human serum albumin before adding PBMCs on top. Monocytes were allowed to transmigrate for $1 \mathrm{~h}$ (unless otherwise stated in the figure legends). Transmigration was arrested by washing with cold PBS to remove nonadherent cells, followed by an overnight fixation with $2.5 \%$ glutaraldehyde in $0.1 \mathrm{M}$ sodium cacodylate buffer, $\mathrm{pH}$ 7.4. Monocyte TEM was quantitated using Nomarski optics, as described previously (5). Typically, we score all of the leukocytes in each of several random fields for each monolayer until at least 100 leukocytes have been counted, with six replicate monolayers for each condition. Therefore, in each experiment, data for each condition are derived from interactions of $>600$ leukocytes with six different monolayers. Transmigration data are expressed as the percentage of total monocytes associated with the HUVEC monolayer that crossed the endothelial monolayer.

Eluate control. In experiments designed to study the effect of depolymerization of endothelial cell microtubules on TEM, an eluate control was performed to test for an effect of residual drugs on monocytes. In these 
experiments, replicate HUVEC monolayers were subjected to the same conditions as those undergoing treatment with depolymerizing agents. Fresh culture medium was added, and these monolayers were returned to the incubator for $1 \mathrm{~h}$ (or whatever the length of the experiment). The conditioned medium, containing whatever drug had eluted during that time, was collected from these monolayers. PBMCs in the eluate control were resuspended directly in this medium and added to untreated HUVEC monolayers. The leukocytes of the eluate control were thus exposed at time zero to all of the drug that would have eluted over the time course of the experiment.

Endothelial microtubule disruption and PECAM recycling. Recycling experiments were performed as previously described (9). In brief, HUVEC monolayers were incubated in the presence of an Fab fragment of $\mathrm{P} 1.1 \mathrm{mAb}$ for $1 \mathrm{~h}$ at $37^{\circ} \mathrm{C}$. This $\mathrm{mAb}$ binds to PECAM domain five and does not block any known PECAM function. It serves as a marker for PECAM in the cell. HUVECs were then chilled and washed of free antibody, and an excess of an $\mathrm{F}\left(\mathrm{ab}^{\prime}\right)_{2}$ fragment of unlabeled goat anti-mouse IgG was added to the cells. After $1 \mathrm{~h}$ of incubation on ice, unbound antibody was washed away, and a rhodamine-conjugated $\mathrm{F}\left(\mathrm{ab}^{\prime}\right)_{2}$ fragment of the same goat anti-mouse antibody was premixed with incubation medium containing either $10 \mu \mathrm{M}$ DCN or DMSO at the concentration used to dissolve DCN. After $1 \mathrm{~h}$ of incubation on ice, cells were placed at $37^{\circ} \mathrm{C}$ and incubated for different time periods, as shown in the figures. Cells were chilled, washed, and fixed in freshly prepared $2 \%$ paraformaldehyde for $10 \mathrm{~min}$ at room temperature.

In experiments to measure the effect of microtubule or actin filament perturbation on recycling of PECAM, HUVEC monolayers were incubated with Fab P1.1 antibody for $1 \mathrm{~h}$ at $37^{\circ} \mathrm{C}$, and $10 \mu \mathrm{M}$ DCN, $10 \mu \mathrm{M}$ taxol, $2 \mu \mathrm{M}$ nocodazole, $400 \mathrm{nM}$ jasplakinolide, or DMSO at the concentration used to dissolve each of the drugs used was added to the P1.1-containing incubation medium. After $1 \mathrm{~h}$ of incubation at $37^{\circ} \mathrm{C}$, cells were chilled and washed, and the remaining steps of the recycling experiment were performed as described in the presence or absence of each drug.

In experiments to examine targeted recycling of membrane from the LBRC during diapedesis, the monolayers were prepared as described in this section. Purified leukocytes were resuspended in the rhodamine-labeled $\mathrm{F}\left(\mathrm{ab}^{\prime}\right)_{2}$ secondary antibody and allowed to settle on top of the HUVEC monolayers on ice. When the monolayers were returned to $37^{\circ} \mathrm{C}$, transmigration and recycling resumed. The co-cultures were washed and fixed as described in this section.

Microscopy and image processing. Fluorescence microscopy was performed on a confocal microscope (LSM510; Carl Zeiss, Inc.) using a $63 \times 1.4$ numerical aperture oil immersion objective, or on an epifluorescence microscope (Axiovert; Carl Zeiss, Inc.) with a cooled charge-coupled device 12-bit camera (Princeton Instruments) using a $25 \times 1.25$ numerical aperture oil immersion objective. Image processing and quantification were performed using image processing software (MetaMorph; MDS Analytical Technologies).

Quantification of targeted recycling. Images were processed using MetaMorph. Background corrected images were highlighted in pseudocolor, and a one-pixel-wide line (narrower than the width of the junction) was drawn along the junctions that surround the site of diapedesis and across the brightest pixels of the ring of recycled PECAM. Intensities at the selected areas were generated by the software. We calculated the maximum PECAM enrichment by dividing the highest fluorescence intensity determined at the ring by the average intensity obtained at the junctions in the vicinity of the ring. The levels of recycling PECAM fluorescence away from the areas of leukocyte contact were found empirically to be the same in control and DCN-treated cells (Fig. 3 C).

Calcium flux assay. HUVEC monolayers were treated with $10 \mu \mathrm{M}$ DCN or DMSO (control) for $1 \mathrm{~h}$ at $37^{\circ} \mathrm{C}$, and then washed and incubated in the dark for $30 \mathrm{~min}$ at $37^{\circ} \mathrm{C}$ with $10 \mu \mathrm{M}$ Fluo3/AM (Invitrogen) in M199 containing $0.025 \%$ pluronic acid, $2.5 \mathrm{mM}$ probenecid, $0.5 \%$ human serum albumin, and $20 \mathrm{mM}$ Hepes. The monolayers were washed twice and incubated in the same buffer without Fluo3 or pluronic acid for $20 \mathrm{~min}$ at room temperature to allow hydrolysis of the dye ester. The culture dishes were then placed on a heated microscope stage. Fluorescent images were acquired every $5 \mathrm{~s}$ before and after the addition of $10 \mu \mathrm{M}$ histamine. Quantitative analysis of the images was performed with MetaMorph software. In brief, after background subtraction, the averaged intensity per field was measured for each time point, and the relative intensities per field for each time point were plotted.

Immuno-EM. Horseradish peroxidase (HRP) was conjugated to P1.1 IgG, as previously described. (9). HUVEC monolayers plated on fibronectincoated coverslip dishes were first loaded with HRP-P1.1 mAb directed against PECAM, and then exposed to control vehicle or $10 \mu \mathrm{M} D C N$ for $1 \mathrm{~h}$ at $37^{\circ} \mathrm{C}$ in the presence of HRP-P1.1 mAb. They were subsequently rinsed in cold PBS and fixed in $4 \%$ glutaraldehyde (EM Sciences) in $0.1 \mathrm{M}$ sodium cacodylate buffer for $15 \mathrm{~min}$. Cells were rinsed, and a mixture of diaminobenzidine and $\mathrm{H}_{2} \mathrm{O}_{2}$ was added to the cells to visualize PECAM staining according to the method of Graham and Karnovsky (44). The cells were postfixed in $4 \%$ glutaraledehyde and embedded in plastic, and en face sections were cut and examined on an electron microscope (JSM 100CX II; JEOL).

Stereologic analysis. Electron micrographs of representative randomly selected en face sections from HUVEC monolayers incubated at $37^{\circ} \mathrm{C}$ with HRP-P1.1 in the presence or absence of DCN were opened in Photoshop (Adobe) and overlaid with grids. Quantitation was performed as previously described (9) by standard stereologic techniques. In brief, the number of times the random lines crossed DAB-labeled internal membrane compartments or junctional membrane were recorded. The ratio is proportional to their relative surface area.

Microinjection of endothelial cells. HUVECs grown to confluence on coverslip dishes were incubated with TNF- $\alpha$ for at least $4 \mathrm{~h}$, during which time they were pressure microinjected with HD IgG (anti-kinesin motor domain) or purified nonimmune rabbit IgG prepared in $\mathrm{HKCl}(10 \mathrm{mM}$ Hepes, $140 \mathrm{mM} \mathrm{KCl}, \mathrm{pH} 7.4$ ) at a concentration of $20 \mathrm{mg} / \mathrm{ml}$ using a micromanipulator (MMN-1; Narishige), as previously described (45). The control $\operatorname{IgG}$ serves as a control for nonspecific damage to the cells caused by the volume of material injected and the presence of a high concentration of exogenous protein. After injection, cells were maintained at $37^{\circ} \mathrm{C}$ for $1 \mathrm{~h}$ before any manipulations involving antibody (e.g., for targeted recycling experiments), and $3 \mathrm{~h}$ before addition of leukocytes in the continued presence of TNF- $\alpha$.

Statistics. All data were analyzed by pairwise comparison using a two-tailed $t$ test assuming unequal variances, with the Bonferroni correction for multiple comparisons as appropriate.

Online supplemental material. Fig. S1 demonstrates that nocodazole depolymerizes microtubules without disrupting endothelial cell junctions. Fig. S2 shows that taxol treatment of HUVECs induces bundling and stabilization of microtubules without disrupting endothelial borders. Fig. S3 shows that microtubule stabilization by taxol and actin filament stabilization by jasplakinolide do not affect monocyte adhesion, but taxol selectively blocks TEM. Fig. S4 shows that taxol does not affect constitutive recycling of membrane from the LBRC. Fig. S5 shows that the DCN treatment used in our experiments does not result in redistribution of mitochondria, endoplasmic reticulum, or calcium flux in response to histamine in the treated endothelial cells, and that microinjection of control and HD antibody do not affect the distribution of intermediate filaments. Fig. S6 demonstrates that the anti-kinesin motor domain antibody used in our experiments, but not control $\operatorname{IgG}$, prevents centrifugal redistribution of mitochondria along microtubules. Fig. S7 shows that microinjection of control IgG does not affect the expression or distribution of PECAM or ICAM-1 in HUVEC monolayers. Table S1 lists the kinesin family members expressed in our cultured HUVECs. Online supplemental material is available at http://www.jem .org/cgi/content/full/jem.20072328/DC1. 
The authors wish to thank Ronald Liebman for excellent technical assistance and Lee Cohen-Gould for expert assistance with electron microscopy. This work was supported by National Heart, Lung, and Blood Institute grants R01 HL046489 and R37 HL064774 to W.A. Muller, and American Cancer Society grant RSG-06-142-01-CSM to G. Kreitzer.

The authors have no conflicting financial interests.

\section{Submitted: 30 October 2007}

Accepted: 12 March 2008

\section{REFERENCES}

1. Muller, W.A. 2003. Leukocyte-endothelial-cell interactions in leukocyte transmigration and the inflammatory response. Trends Immunol. 24:326-333.

2. Rao, R.M., L. Yang, G. Garcia-Cardena, and F.W. Luscinskas. 2007. Endothelial-dependent mechanisms of leukocyte recruitment to the vascular wall. Circ. Res. 101:234-247.

3. Muller, W.A., C.M. Ratti, S.L. McDonnell, and Z.A. Cohn. 1989. A human endothelial cell-restricted, externally disposed plasmalemmal protein enriched in intercellular junctions. J. Exp. Med. 170:399-414.

4. Newman, P.J., M.C. Berndt, J. Gorski, G.C. White II, S. Lyman, C. Paddock, and W.A. Muller. 1990. PECAM-1 [CD31] cloning and relation to adhesion molecules of the immunoglobulin gene superfamily. Science. 247:1219-1222.

5. Muller, W.A., S.A. Weigl, X. Deng, and D.M. Phillips. 1993. PECAM1 is required for transendothelial migration of leukocytes. J. Exp. Med. 178:449-460.

6. Liao, F., H.K. Huynh, A. Eiroa, T. Greene, E. Polizzi, and W.A. Muller. 1995. Migration of monocytes across endothelium and passage through extracellular matrix involve separate molecular domains of PECAM-1. J. Exp. Med. 182:1337-1343.

7. Liao, F., J. Ali, T. Greene, and W.A. Muller. 1997. Soluble domain 1 of platelet-endothelial cell adhesion molecule (PECAM) is sufficient to block transendothelial migration in vitro and in vivo. J. Exp. Med. 185:1349-1357.

8. Liao, F., A.R. Schenkel, and W.A. Muller. 1999. Transgenic mice expressing different levels of soluble platelet/endothelial cell adhesion molecule- $\operatorname{IgG}$ display distinct inflammatory phenotypes. J. Immunol. 163:5640-5648.

9. Mamdouh, Z., X. Chen, L.M. Pierini, F.R. Maxfield, and W.A. Muller. 2003. Targeted recycling of PECAM from endothelial cell surface-connected compartments during diapedesis. Nature. 421:748-753.

10. Feng, D., J.A. Nagy, J. Hipp, H.F. Dvorak, and A.M. Dvorak. 1996. Vesiculo-vacuolar organelles and the regulation of venule permeability to macromolecules by vascular permeability factor, histamine, and serotonin. J. Exp. Med. 183:1981-1986.

11. Wozniak, M.J., R. Milner, and V. Allan. 2004. N-terminal kinesins: many and various. Traffic. 5:400-410.

12. Schenkel, A.R., Z. Mamdouh, X. Chen, R.M. Liebman, and W.A. Muller. 2002. CD99 plays a major role in the migration of monocytes through endothelial junctions. Nat. Immunol. 3:143-150.

13. Samson, F., J.A. Donoso, I. Heller-Bettinger, D. Watson, and R.H. Himes. 1979. Nocodazole action on tubulin assembly, axonal ultrastructure and fast axoplasmic transport. J. Pharmacol. Exp. Ther. 208:411-417.

14. Green, K.J., and R.D. Goldman. 1983. The effects of taxol on cytoskeletal components in cultured fibroblasts and epithelial cells. Cell Motil. 3:283-305.

15. Campello, S., R.A. Lacalle, M. Bettella, S. Manes, L. Scorrano, and A. Viola. 2006. Orchestration of lymphocyte chemotaxis by mitochondrial dynamics. J. Exp. Med. 203:2879-2886.

16. Wu, S., H. Chen, M.F. Alexeyev, J.A. King, T.M. Moore, T. Stevens, and R.D. Balczon. 2007. Microtubule motors regulate ISOC activation necessary to increase endothelial cell permeability. J. Biol. Chem. 282:34801-34808.

17. Huang, A.J., J.E. Manning, T.M. Bandak, M.C. Ratau, K.R. Hanser, and S.C. Silverstein. 1993. Endothelial cell cytosolic free calcium regulates neutrophil migration across monolayers of endothelial cells. J. Cell Biol. 120:1371-1380.
18. Bird, I.N., J.H. Spragg, A.H. Ager, and N. Matthews. 1993. Studies of lymphocyte transendothelial migration: analysis of migrated cell phenotypes with regard to CD31 (PECAM-1), CD45RA and CD45RO. Immunology. 80:553-560.

19. Muller, W.A. 2001. Migration of leukocytes across endothelial junctions: some concepts and controversies. Microcirculation. 8:181-193.

20. Gyoeva, F.K., and V.I. Gelfand. 1991. Coalignment of vimentin intermediate filaments with microtubules depends on kinesin. Nature. 353:445-448.

21. Wright, B.D., M. Terasaki, and J.M. Scholey. 1993. Roles of kinesin and kinesin-like proteins in sea urchin embryonic cell division: evaluation using antibody microinjection. J. Cell Biol. 123:681-689.

22. Nangaku, M., R. Sato-Yoshitake, Y. Okada, Y. Noda, R. Takemura, H. Yamazaki, and N. Hirokawa. 1994. KIF1B, a novel microtubule plus end-directed monomeric motor protein for transport of mitochondria. Cell. 79:1209-1220.

23. Rodionov, V.I., F.K. Gyoeva, E. Tanaka, A.D. Bershadsky, J.M. Vasiliev, and V.I. Gelfand. 1993. Microtubule-dependent control of cell shape and pseudopodial activity is inhibited by the antibody to kinesin motor domain. J. Cell Biol. 123:1811-1820.

24. Tanaka, Y., Y. Kanai, Y. Okada, S. Nonaka, S. Takeda, A. Harada, and N. Hirokawa. 1998. Targeted disruption of mouse conventional kinesin heavy chain, kif5B, results in abnormal perinuclear clustering of mitochondria. Cell. 93:1147-1158.

25. Huang, M.T., K.Y. Larbi, C. Scheiermann, A. Woodfin, N. Gerwin, D.O. Haskard, and S. Nourshargh. 2006. ICAM-2 mediates neutrophil transmigration in vivo: evidence for stimulus specificity and a role in PECAM-1-independent transmigration. Blood. 107:4721-4727.

26. Ostermann, G., K.S.C. Weber, A. Zernecke, A. Schroder, and C. Weber. 2002. JAM-1 is a ligand of the $\beta_{2}$ integrin LFA-1 involved in transendothelial migration of leukocytes. Nat. Immunol. 3:151-158.

27. Wegmann, F., B. Petri, A.G. Khandoga, C. Moser, A. Khandoga, S. Volkery, H. Li, I. Nasdala, O. Brandau, R. Fassler, et al. 2006. ESAM supports neutrophil extravasation, activation of Rho, and VEGF-induced vascular permeability. J. Exp. Med. 203:1671-1677.

28. Reymond, N., A.M. Imbert, E. Devilard, S. Fabre, C. Chabannon, L. Xerri, C. Farnarier, C. Cantoni, C. Bottino, A. Moretta, et al. 2004 DNAM-1 and PVR regulate monocyte migration through endothelial junctions. J. Exp. Med. 199:1331-1341.

29. Barreiro, O., M. Yanez-Mo, J.M. Serrador, M.C. Montoya, M. VicenteManzanares, R. Tejedor, H. Furthmayr, and F. Sanchez-Madrid. 2002 Dynamic interaction of VCAM-1 and ICAM-1 with moesin and ezrin in a novel endothelial docking structure for adherent leukocytes. J. Cell Biol. 157:1233-1245.

30. Carman, C.V., and T.A. Springer. 2004. A transmigratory cup in leukocyte diapedesis both through individual vascular endothelial cells and between them. J. Cell Biol. 167:377-388.

31. Shaw, S.K., S. Ma, M.B. Kim, R.M. Rao, C.U. Hartman, R.M. Froio, L. Yang, T. Jones, Y. Liu, A. Nusrat, et al. 2004. Coordinated redistribution of leukocyte LFA-1 and endothelial cell ICAM-1 accompany neutrophil transmigration. J. Exp. Med. 200:1571-1580.

32. Schenkel, A.R., Z. Mamdouh, and W.A. Muller. 2004. Locomotion of monocytes on endothelium is a critical step during extravasation. Nat. Immunol. 5:393-400.

33. Phillipson, M., B. Heit, P. Colarusso, L. Liu, C.M. Ballantyne, and P. Kubes. 2006. Intraluminal crawling of neutrophils to emigration sites: a molecularly distinct process from adhesion in the recruitment cascade. $J$. Exp. Med. 203:2569-2575.

34. Bixel, G., S. Kloep, S. Butz, B. Petri, B. Engelhardt, and D. Vestweber. 2004. Mouse CD99 participates in $\mathrm{T}$ cell recruitment into inflamed skin. Blood. 104:3205-3213.

35. Bixel, M.G., B. Petri, A.G. Khandoga, A. Khandoga, K. WolburgBuchholz, H. Wolburg, S. Marz, F. Krombach, and D. Vestweber. 2007. A CD99-related antigen on endothelial cells mediates neutrophil but not lymphocyte extravasation in vivo. Blood. 109:5327-5336.

36. Randolph, G.J., S. Beaulieu, S. Lebecque, R.M. Steinman, and W.A. Muller. 1998. Differentiation of monocytes into dendritic cells in a model of transendothelial trafficking. Science. 282:480-483. 
37. Randolph, G.J., K. Inaba, D.F. Robbiani, R.M. Steinman, and W.A. Muller. 1999. Differentiation of phagocytic monocytes into lymph node dendritic cells in vivo. Immunity. 11:753-761.

38. Kitayama, J., A. Hidemura, H. Saito, and H. Nagawa. 2000. Shear stress affects migration behavior of polymorphonuclear cells arrested on endothelium. Cell. Immunol. 203:39-46.

39. Cuvelier, S.L., and K.D. Patel. 2001. Shear-dependent eosinophil transmigration on interleukin 4-stimulated endothelial cells: a role for endothelium-associated eotaxin-3. J. Exp. Med. 194:1699-1709.

40. Allport, J.R., W.A. Muller, and F.W. Luscinskas. 2000. Monocytes induce reversible focal changes in vascular endothelial cadherin complex during transendothelial migration under flow. J. Cell Biol. 148:203-216.

41. Shaw, S.K., P.S. Bamba, B.N. Perkins, and F.W. Luscinskas. 2001. Real-time imaging of vascular endothelial-cadherin during leukocyte transmigration across endothelium. J. Immunol. 167:2323-2330.
42. Thompson, R.D., K.E. Noble, K.Y. Larbi, A. Dewar, G.S. Duncan, T.W. Mak, and S. Nourshargh. 2001. Platelet-endothelial cell adhesion molecule-1 (PECAM-1)-deficient mice demonstrate a transient and cytokine-specific role for PECAM-1 in leukocyte migration through the perivascular basement membrane. Blood. 97:1854-1860.

43. Muller, W.A., and S. Weigl. 1992. Monocyte-selective transendothelial migration: dissection of the binding and transmigration phases by an in vitro assay. J. Exp. Med. 176:819-828.

44. Graham, R.C., Jr., and M.J. Karnovsky. 1966. The early stages of absorption of injected horseradish peroxidase in the proximal tubules of mouse kidney: ultrastructural cytochemistry by a new technique. J. Histochem. Cytochem. 14:291-302.

45. Kreitzer, G., A. Marmorstein, P. Okamoto, R. Vallee, and E. RodriguezBoulan. 2000. Kinesin and dynamin are required for post-Golgi transport of a plasma-membrane protein. Nat. Cell Biol. 2:125-127. 